\title{
Diabetes diagnosis and nutrition facts label use among US adults, 2005-2010
}

\section{Ruopeng An*}

Department of Kinesiology and Community Health, College of Applied Health Sciences, University of Illinois at Urbana-Champaign, 1206 S 4th Street, Champaign, IL 61820, USA

Submitted 22 March 2015: Final revision received 1 September 2015: Accepted 25 September 2015: First published online 20 0ctober 2015

\begin{abstract}
Objective: To assess the role of diabetes diagnosis as a potential teachable moment in nutrition facts label use among US adults.

Design: Logistic regression analyses were conducted to examine the relationship between diabetes diagnosis status (diagnosed diabetes, undiagnosed diabetes, diagnosed prediabetes, undiagnosed prediabetes, no diabetes or prediabetes) and self-reported nutrition facts label use, adjusted by individual characteristics and survey design.

Setting: Study sample came from the National Health and Nutrition Examination Survey 2005-2010 waves.

Subjects: A total of 5110 US adults aged 20 years and older were included in the analyses. Diabetes/prediabetes was identified by fasting plasma glucose and glycated $\mathrm{Hb}$ testing.

Results: People with diagnosed diabetes/prediabetes were substantially more likely to report nutrition facts label use when making daily food purchase decisions compared with those with undiagnosed diabetes/prediabetes, whereas the prevalence of nutrition facts label use was similar between people with undiagnosed diabetes/prediabetes and those without diabetes/prediabetes. The adjusted prevalence ( $95 \% \mathrm{CI}$ ) of any and regular nutrition facts label use was $85.93(82.91,88.95) \%$ and $55.60(50 \cdot 04,61 \cdot 16) \%$ among those with diagnosed diabetes, respectively, in comparison to 71.50 (59.64, 83.37) \% and 32.88 (19.11, $46.65) \%$ among those with undiagnosed diabetes. Analogously, the adjusted prevalence $(95 \% \mathrm{CI})$ of any and regular nutrition facts label use was $81 \cdot 16(75 \cdot 27$, $87.06) \%$ and $45.28(37 \cdot 28,53 \cdot 29) \%$ among those with diagnosed prediabetes, respectively, in comparison to $72.83(68.06,77.59) \%$ and $39.95(34.02,45 \cdot 89) \%$ among those with undiagnosed prediabetes.

Conclusions: As a potential teachable moment, diabetes diagnosis may positively impact nutrition facts label use and motivate diabetic patients to manage their condition through making healthier food choices.
\end{abstract}

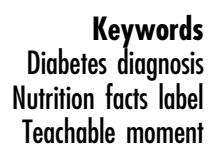

As a leading cause of disability and premature mortality in the USA and worldwide, diabetes can result in serious health complications including heart disease, blindness, kidney failure and lower-extremity amputations ${ }^{(1)}$. In 2012, an estimated 28.9 million American adults aged 20 years and above had diagnosed or undiagnosed diabetes and an additional 86 million had prediabetes ${ }^{(2)}$. A healthy diet is a crucial regime for diabetes management ${ }^{(3)}$. Patients with diabetes should limit their consumption of foods high in sugar, salt or fat; carefully monitor the time and quantity of carbohydrate intake; consume a variety of fruits, vegetables and whole-grain foods on a daily basis; and eat smaller portions and spread out their meals over the day. The nutrition facts label is an essential source for consumers to obtain nutrition-related information on food products and serves as a population-level policy intervention with unparalleled reach ${ }^{(4)}$. Nutrition facts labels may help patients with diabetes manage their condition through making healthier food choices.

A long line of research documents consumers' understanding and use of nutrition facts labels, as well as their impact on dietary behaviour ${ }^{(5-8)}$. A substantial proportion of US consumers report regular use of nutrition facts labels to guide their selection of food products ${ }^{(5-8)}$. The perception on the credibility of nutrition facts labels appears high and there is a consistent association between nutrition facts label use and diet quality ${ }^{(5-8)}$. The American Diabetes Association recommends that patients "use the 
information on the nutrition facts label to compare foods and make better choices ${ }^{(9)}$. However, little is known regarding the prevalence of nutrition facts label use among the diabetic population and whether that differs between people with and without diabetes and across diagnosis status. Behavioural theories and interventions emphasize the importance of cues in prompting motivation for behaviour change ${ }^{(10)}$. A specific type of cue, labelled a 'teachable moment', describes a naturally occurring health or life event that motivates individuals to spontaneously engage in risk-reducing health-promoting behaviour ${ }^{(11)}$. While chronic illnesses like diabetes are a major threat to health and quality of life, disease diagnosis itself could serve as a critical teachable moment for patients to initiate behaviour change $\mathrm{e}^{(12-15)}$. At a diagnosis of diabetes or prediabetes, patients may be particularly concerned about their health status and willing to accept recommendations on behaviour modification in an effort to effectively manage their diabetic condition. Physicians and other nutrition/health professionals may seize this window of opportunity to provide patients with tailored messages and advice regarding food choices, physical activity and other health behaviours. Nutrition education programmes may also take advantage of diabetes diagnosis to improve effectiveness, reduce cost and adapt to a larger population.

The impact of chronic condition diagnosis on behaviour modification as a teachable moment has been consistently documented in smoking cessation ${ }^{(16-24)}$, but findings remain mixed for other types of health behaviours such as drinking and physical activity ${ }^{(19-22,25-27)}$. To our knowledge, the present study serves as the first attempt to evaluate the role of diabetes diagnosis as a potential teachable moment in nutrition facts label use. If diabetes/ prediabetes diagnosis qualifies for a teachable moment, we would expect that patients with diagnosed diabetes/ prediabetes pay more attention to their daily diet in an effort to better manage their diabetic condition compared with their counterparts who suffer from the same illness but are unaware of it (i.e. people with undiagnosed diabetes/ prediabetes). On the other hand, we would expect the grocery shopping and diet behaviours among those with undiagnosed diabetes/prediabetes to be similar to those of people without diabetes/prediabetes, since the former group may not pay additional attention to their daily diet due to their unawareness of their diabetic condition. Therefore, we hypothesized that: (i) people with diagnosed diabetes/prediabetes are more likely to use nutrition facts labels in guiding their daily food purchases compared with people with undiagnosed diabetes/prediabetes; and (ii) the prevalence of nutrition facts label use does not differ between people with undiagnosed diabetes/prediabetes and people without diabetes/prediabetes. Using data from a nationally representative repeated cross-sectional survey, we tested this hypothesis by examining the relationship between diabetes diagnosis status and nutrition facts label use among US adults aged 20 years and above.

\section{Methods}

\section{Survey setting and participants}

Individual-level data came from the National Health and Nutrition Examination Survey (NHANES) 2005-2006, 2007-2008 and 2009-2010 waves. NHANES is a programme of studies conducted by the National Center for Health Statistics to assess the health and nutritional status of children and adults. The programme began in the early 1960s and has periodically conducted separate surveys focusing on different population groups or health topics. Since 1999, NHANES has been conducted continuously in two-year cycles and has a changing focus on a variety of health and nutrition measurements. A multistage probability sampling design is used to select participants representative of the civilian, non-institutionalized US population. Certain population subgroups are oversampled to increase the reliability and precision of health status indicator estimates for these groups.

\section{Use of nutrition facts label}

In a survey respondent's home, a trained interviewer presented the respondent with a hand card and asked the question: 'Here is an example of a food label. This part of the food label is called the Nutrition Facts panel. How often do you use the Nutrition Facts panel when deciding to buy a food product? Would you say always, most of the time, sometimes, rarely, or never?' Any use of nutrition facts label is defined by grouping the answers of 'always', 'most of the time' and 'sometimes', with the reference group consisting of the answers of 'rarely' and 'never'. Regular use of nutrition facts label is defined by the answers of 'always' and 'most of the time', with the reference group consisting of the answers of 'sometimes', 'rarely' and 'never'.

\section{Diabetes measures}

Following the definitions adopted in the Centers for Disease Control and Prevention weekly report ${ }^{(28)}$, we classified survey respondents into five mutually exclusive categories based on their self-reported diabetes diagnosis and laboratory test result: (i) diagnosed diabetes; (ii) diagnosed prediabetes; (iii) undiagnosed diabetes; (iv) undiagnosed prediabetes; and (v) no diabetes/prediabetes. Diagnosed diabetes was identified by the answer of 'yes' to the interview question 'Have you ever been told by a doctor or health professional that you have diabetes or sugar diabetes?' Diagnosed prediabetes was identified by the answer of 'yes' to the interview question 'Have you ever been told by a doctor or other health professional that you have any of the following: prediabetes, impaired fasting glucose, impaired glucose tolerance, borderline diabetes or that your blood sugar is higher than normal but not high enough to be called diabetes or sugar diabetes?' and the answer of 'prediabetes' when asked whether one had diabetes. Undiagnosed diabetes was identified by having fasting plasma glucose $(\mathrm{FPG}) \geq 126 \mathrm{mg} / \mathrm{dl}$ or glycated $\mathrm{Hb}$ 
(HbA1c) $\geq 6.5 \%$ but reporting absence of diagnosed diabetes. Undiagnosed prediabetes was identified by having $100 \mathrm{mg} / \mathrm{dl} \leq \mathrm{FPG}<126 \mathrm{mg} / \mathrm{dl}$ or $5.7 \% \leq \mathrm{HbA} 1 \mathrm{c}<6.5 \%$ but reporting absence of diagnosed diabetes/prediabetes. Respondents with no diabetes/prediabetes were identified by having $\mathrm{FPG}<100 \mathrm{mg} / \mathrm{dl}$ and $\mathrm{HbA} 1 \mathrm{c}<5.7 \%$ and reporting absence of diagnosed diabetes/prediabetes.

Of the 7763 non-pregnant survey respondents aged 20 years and above assigned to a morning fasting session, the following participants were excluded from the analyses: missing test results for FPG or HbA1c, $n$ 431; missing values for self-reported diabetes/prediabetes diagnosis, $n$ 156; missing values for nutrition facts label use, $n$ 1157; and missing values for other individual characteristics, $n$ 909. The remaining 5110 survey respondents were included in the analysis.

\section{Individual characteristics}

The following individual characteristics were controlled for in the regression analysis: a dichotomous variable for female (male in the reference group); three dichotomous variables for age groups (35-49 years of age, 50-64 years of age and 65 years of age and above, with 20-34 years of age in the reference group); three dichotomous variables for race/ ethnicity (non-Hispanic African American, non-Hispanic other race/multi-race and Hispanic, with non-Hispanic white in the reference group); a dichotomous variable for college education and above (with high school or lower education in the reference group); two dichotomous variables for marital status (divorced/separated/widowed and never married, with married in the reference group); two dichotomous variables for household income level $(130 \% \leq$ income to poverty ratio (IPR) $<300 \%$ and IPR $\geq 300 \%$, with IPR $<130 \%$ in the reference group); a dichotomous variable for obesity (non-obese people in the reference group); a dichotomous variable for ever or current smoker (never smoker in the reference group); a dichotomous variable for good or excellent self-rated health (poor or fair self-rated health in the reference group); a dichotomous variable for any public or private health insurance coverage (no health insurance in the reference group); four dichotomous variables for each of the chronic conditions of arthritis, CHD, stroke and cancer; and two dichotomous variables for NHANES 2007-2008 and 2009-2010 waves (2005-2006 wave in the reference group).

Besides the common individual demographic (sex, age, race/ethnicity) and socio-economic (education, marital status and income level) variables, we also intended to control risky behaviour (smoking) and health/disease status (self-rated health and chronic illnesses) in the regression analysis, because these factors may confound in our estimated relationship between diabetes diagnosis ('exposure') and nutrition facts label use ('outcome') by correlating with both the exposure and the outcome. We further included health insurance status in the model, because the uninsured could be less likely to use healthcare services and have a diabetes diagnosis.

\section{Statistical analyses}

Logistic regression analyses were conducted to examine the relationship between diabetes diagnosis status and nutrition facts label use among US adults, adjusted by individual characteristics. Separate regression models were estimated for the two dependent variables: a dichotomous variable for any use of nutrition facts labels and a dichotomous variable for regular use of nutrition facts labels. The key independent variables were four dichotomous variables for having diagnosed diabetes, diagnosed prediabetes, undiagnosed diabetes and undiagnosed prediabetes, with those reporting no diabetes/prediabetes in the reference group. We calculated the adjusted prevalence of nutrition facts label use by diabetes status based on logistic regression estimates. The differences in the adjusted prevalence of nutrition facts label use across diabetes status were tested using Wald tests.

All statistical analyses were conducted using the statistical software package Stata 14.0 SE version. The NHANES sampling design was incorporated in both descriptive statistics and regression analyses.

\section{Human subjects review}

NHANES was approved by the National Center for Health Statistics Research Ethics Review Board. The present analysis used NHANES de-identified public data and was deemed exempt by the University of Illinois at UrbanaChampaign Institutional Review Board.

\section{Results}

Table 1 reports nutrition facts label use, diabetes diagnosis status and other individual characteristics of adult survey respondents in the NHANES 2005-2010 waves. Among a total of 5110 respondents, the population-weighted prevalence of any nutrition facts label use was $72.59 \%$ and that of regular nutrition facts label use was $41.54 \%$. Nearly a quarter (23.00\%) of survey respondents had diabetes or prediabetes, where undiagnosed prediabetes accounted for $10.09 \%$, undiagnosed diabetes for $0.86 \%$, diagnosed prediabetes for $4.06 \%$ and diagnosed diabetes accounted for $7.99 \%$.

Table 2 reports nutrition facts label use by diabetes diagnosis status. The population-weighted prevalence of any nutrition facts label use was substantially higher among those with diagnosed prediabetes $(81.90 \%)$ and diabetes $(82.50 \%)$ in comparison to those with undiagnosed prediabetes $(71 \cdot 90 \%)$ and diabetes $(62 \cdot 18 \%)$. Analogously, the population-weighted prevalence of regular nutrition facts label use was substantially higher among those with diagnosed prediabetes (48.93\%) and diabetes (54.68\%) in comparison to those with undiagnosed prediabetes (41.32\%) and diabetes (30.60\%). 
Table 1 Individual characteristics of adult NHANES 2005-2010 participants

\begin{tabular}{|c|c|c|}
\hline Individual characteristic & Percentage & $95 \% \mathrm{Cl}$ \\
\hline \multicolumn{3}{|l|}{ Nutrition facts label use status } \\
\hline Any use & 72.59 & $71.09,74.10$ \\
\hline Regular use & 41.54 & $39.80,43.27$ \\
\hline \multicolumn{3}{|l|}{ Diabetes status } \\
\hline No prediabetes/diabetes & 77.00 & $75.47,78.52$ \\
\hline Undiagnosed prediabetes & 10.09 & $9.20,10.98$ \\
\hline Undiagnosed diabetes & 0.86 & $0.57,1.14$ \\
\hline Diagnosed prediabetes & 4.06 & $3.42,4.71$ \\
\hline Diagnosed diabetes & 7.99 & $7.01,8.98$ \\
\hline \multicolumn{3}{|l|}{ Sex } \\
\hline Female & 51.98 & $50 \cdot 62,53 \cdot 35$ \\
\hline \multicolumn{3}{|l|}{ Age group } \\
\hline $20-34$ years of age & 26.94 & $24.97,28.92$ \\
\hline $35-49$ years of age & 31.67 & $29.27,34.07$ \\
\hline $50-64$ years of age & 24.65 & $22 \cdot 69,26 \cdot 62$ \\
\hline 65 years of age and above & $16 \cdot 74$ & $15 \cdot 16,18 \cdot 31$ \\
\hline \multicolumn{3}{|l|}{ Race/ethnicity } \\
\hline White, non-Hispanic & $72 \cdot 78$ & $69 \cdot 40,76 \cdot 16$ \\
\hline African American, non-Hispanic & $10 \cdot 67$ & $8.77,12.57$ \\
\hline Other race/multi-race, non-Hispanic & 5.49 & $4.42,6.56$ \\
\hline Hispanic & 11.06 & $9.04,13.08$ \\
\hline \multicolumn{3}{|l|}{ Education level } \\
\hline College education and above & 60.00 & $56.98,63.01$ \\
\hline \multicolumn{3}{|l|}{ Marital status } \\
\hline Married & 65.95 & $63.93,67.98$ \\
\hline Divorced/separated/widowed & 17.68 & $16.50,18.86$ \\
\hline Never married & $16 \cdot 37$ & $14.40,18.33$ \\
\hline \multicolumn{3}{|l|}{ Household income level } \\
\hline IPR $<130 \%$ & $18 \cdot 25$ & $16 \cdot 24,20 \cdot 26$ \\
\hline $130 \% \leq \mathrm{IPR}<300 \%$ & 28.92 & $26 \cdot 46,31 \cdot 38$ \\
\hline IPR $\geq 300 \%$ & 52.83 & $49.90,55.75$ \\
\hline \multicolumn{3}{|l|}{ Obesity status } \\
\hline Obese $\left(\mathrm{BMI} \geq 30.0 \mathrm{~kg} / \mathrm{m}^{2}\right)$ & 34.83 & $32 \cdot 83,36 \cdot 84$ \\
\hline \multicolumn{3}{|l|}{ Smoking status } \\
\hline Former or current smoker & $46 \cdot 27$ & $43.76,48.78$ \\
\hline \multicolumn{3}{|l|}{ Current health status } \\
\hline Good or excellent health & 83.95 & $82.63,85.27$ \\
\hline \multicolumn{3}{|l|}{ Health insurance status } \\
\hline With health insurance & $82 \cdot 32$ & $80 \cdot 34,84 \cdot 30$ \\
\hline \multicolumn{3}{|l|}{ Chronic condition } \\
\hline Arthritis & 26.05 & $24.00,28 \cdot 11$ \\
\hline Coronary artery disease & 3.35 & $2.80,3.89$ \\
\hline Stroke & $2 \cdot 73$ & $2 \cdot 10,3 \cdot 35$ \\
\hline Cancer & $8 \cdot 68$ & $7.74,9.62$ \\
\hline \multicolumn{3}{|l|}{ Survey wave } \\
\hline 2005-2006 & 37.48 & $34.00,40.96$ \\
\hline $2007-2008$ & 30.83 & $26.74,34.92$ \\
\hline 2009-2010 & 31.69 & $28.53,34.86$ \\
\hline
\end{tabular}

NHANES, National Health and Nutrition Examination Survey; IPR, income to poverty ratio.

Individual-level data ( $n$ 5110) from NHANES 2005-2010 waves. NHANES sampling design was incorporated in estimating the percentages.

Table 3 reports the estimated odds ratios of nutrition facts label use in logistic regression analyses. After adjusting for individual characteristics, the odds of any and regular nutrition facts label use among people with diagnosed diabetes were $110 \%$ (adjusted odds ratio $(\mathrm{AOR})=$ $2 \cdot 10 ; 95 \% \mathrm{CI}=1 \cdot 62,2 \cdot 73)$ and $94 \%(\mathrm{AOR}=1.94 ; 95 \% \mathrm{CI}$ $1.55,2.43)$ higher, respectively, than among their counterparts without diabetes/prediabetes. In contrast, no difference in the odds of any (AOR $=0 \cdot 86 ; 95 \%$ CI 0.47 , 1.59) and regular nutrition facts label use $(\mathrm{AOR}=0.76$; $95 \%$ CI $0.40,1.46$ ) was found between those with
Table 2 Unadjusted prevalence of nutrition facts label use by diabetes status in NHANES 2005-2010 adult participants

\begin{tabular}{|c|c|c|c|c|}
\hline \multirow[b]{3}{*}{ Diabetes status } & \multicolumn{4}{|c|}{ Nutrition facts label use status } \\
\hline & \multicolumn{2}{|c|}{ Any use } & \multicolumn{2}{|c|}{ Regular use } \\
\hline & $\%$ & $95 \% \mathrm{Cl}$ & $\%$ & $95 \% \mathrm{Cl}$ \\
\hline No prediabetes/diabetes & 69.20 & $67.53,70.88$ & 37.53 & $35.62,39.45$ \\
\hline $\begin{array}{l}\text { Undiagnosed } \\
\text { prediabetes }\end{array}$ & 71.90 & $67 \cdot 24,76 \cdot 56$ & $41 \cdot 32$ & $35 \cdot 49,47 \cdot 16$ \\
\hline Undiagnosed diabetes & $62 \cdot 18$ & $50.51,73.85$ & $30 \cdot 60$ & $20 \cdot 40,40 \cdot 81$ \\
\hline Diagnosed prediabetes & 81.90 & $75 \cdot 50,88.31$ & 48.93 & $40 \cdot 77,57 \cdot 10$ \\
\hline Diagnosed diabetes & 82.50 & $79.09,85.90$ & 54.68 & $50 \cdot 26,59 \cdot 10$ \\
\hline
\end{tabular}

NHANES, National Health and Nutrition Examination Survey.

Individual-level data ( $n$ 5110) from NHANES 2005-2010 waves. Any use of nutrition facts label is defined as using labels sometimes, mostly or always. Regular use of nutrition facts label is defined as mostly or always using labels. NHANES sampling design was incorporated in estimating the percentages.

undiagnosed diabetes and those without diabetes/ prediabetes. The odds of any and regular nutrition facts label use among people with diagnosed prediabetes were $48 \%(\mathrm{AOR}=1 \cdot 48 ; 95 \% \mathrm{CI} 0 \cdot 97,2 \cdot 26)$ and $28 \%(\mathrm{AOR}=$ 1.28; $95 \%$ CI $0.91,1.80$ ) higher, respectively, than among their counterparts without diabetes/prediabetes, although these differences were not statistically significant at $P<0.05$. In contrast, no difference in the odds of any $(\mathrm{AOR}=0.92 ; 95 \% \mathrm{CI} 0.71,1.20)$ and regular nutrition facts label use $(\mathrm{AOR}=1.03 ; 95 \%$ CI $0.80,1.34)$ was found between those with undiagnosed prediabetes and those without diabetes/prediabetes. As for other individual characteristics, females, middle-aged and older adults (50 years of age and above), people with college education and above, with good or excellent self-rated health and covered by health insurance were significantly more likely to use nutrition facts labels compared with males, younger adults, people with high school or lower education, with poor or fair self-rated health and without health insurance coverage.

Table 4 reports the adjusted prevalence of any and regular nutrition facts label use based on the logistic regression estimates. The adjusted prevalence of any nutrition facts label use was substantially higher among those with diagnosed diabetes $(85.93 \%)$ in comparison to undiagnosed diabetes $(71.50 \% ; P<0.001)$; and the adjusted prevalence of any nutrition facts label use was also noticeably higher among those with diagnosed prediabetes $(81.16 \%)$ in comparison to undiagnosed prediabetes ( $72.83 \% ; P<0.05)$. Analogously, the adjusted prevalence of regular nutrition facts label use was substantially higher among those with diagnosed diabetes (55.60\%) in comparison to undiagnosed diabetes $(32.88 \% ; P<0 \cdot 001)$. The adjusted prevalence of regular nutrition facts label use also tended to be higher among those with diagnosed prediabetes $(45.28 \%)$ in comparison to undiagnosed prediabetes $(39.95 \%)$, although the difference was not statistically significant at $P<0 \cdot 05$. 
Table 3 Estimated odds ratios of nutrition facts label use in NHANES 2005-2010 adult participants in logistic regression analyses

\begin{tabular}{|c|c|c|c|c|c|}
\hline \multirow[b]{3}{*}{ Individual characteristic } & \multicolumn{5}{|c|}{ Nutrition facts label use status } \\
\hline & \multicolumn{2}{|c|}{ Any use } & \multicolumn{3}{|c|}{ Regular use } \\
\hline & OR & $95 \% \mathrm{Cl}$ & OR & & $95 \% \mathrm{Cl}$ \\
\hline \multicolumn{6}{|l|}{ Diabetes status } \\
\hline No prediabetes/diabetes (ref.) & \multicolumn{2}{|c|}{1.000} & \multicolumn{3}{|c|}{1.000} \\
\hline Undiagnosed prediabetes & 0.922 & $0.707,1.202$ & 1.032 & & $0.795,1.338$ \\
\hline Undiagnosed diabetes & 0.863 & $0.469,1.590$ & 0.760 & & $0.395,1.459$ \\
\hline Diagnosed prediabetes & 1.482 & $0.974,2.255$ & 1.283 & & $0.913,1.802$ \\
\hline Diagnosed diabetes & $2 \cdot 102^{\star \star \star}$ & $1 \cdot 620,2 \cdot 728$ & $1.942^{\star \star \star}$ & & $1.553,2.428$ \\
\hline \multicolumn{6}{|l|}{ Sex } \\
\hline Male (ref.) & \multicolumn{2}{|c|}{1.000} & \multicolumn{3}{|c|}{1.000} \\
\hline Female & $2 \cdot 628^{\star \star \star}$ & $2 \cdot 151,3 \cdot 210$ & $2.053^{\star \star \star}$ & & $1 \cdot 797,2 \cdot 344$ \\
\hline \multicolumn{6}{|l|}{ Age group } \\
\hline 20-34 years of age (ref.) & \multicolumn{2}{|c|}{1.000} & \multicolumn{3}{|c|}{1.000} \\
\hline $35-49$ years of age & $1.372^{*}$ & $1.054,1.786$ & 1.246 & & $0.990,1.568$ \\
\hline $50-64$ years of age & $1.958^{\star *}$ & $1.336,2.868$ & $1.618^{\star *}$ & & $1 \cdot 225,2 \cdot 137$ \\
\hline 65 years of age and above & $1.744^{\star \star}$ & $1.237,2.458$ & $1.960^{\star \star *}$ & & $1.512,2.541$ \\
\hline \multicolumn{6}{|l|}{ Race/ethnicity } \\
\hline White, non-Hispanic (ref.) & \multicolumn{2}{|c|}{1.000} & \multicolumn{3}{|c|}{1.000} \\
\hline African American, non-Hispanic & $0.751^{\star *}$ & $0.618,0.913$ & 0.885 & & $0.716,1.093$ \\
\hline Other race/multi-race, non-Hispanic & $1 \cdot 160$ & $0.745,1.804$ & 0.971 & & $0.703,1.342$ \\
\hline Hispanic & 0.975 & $0.814,1.168$ & 1.139 & & $0.927,1.398$ \\
\hline \multicolumn{6}{|l|}{ Education level } \\
\hline High school or lower (ref.) & & & & 1.000 & \\
\hline College education and above & $1.814^{\star \star \star}$ & $1.512,2.176$ & $1.630^{\star \star \star}$ & & $1.358,1.956$ \\
\hline Marital status & & & & & \\
\hline Married (ref.) & & & & 1.000 & \\
\hline Divorced/separated/widowed & 0.905 & $0.692,1.183$ & 1.032 & & $0.825,1.289$ \\
\hline Never married & 1.014 & $0.769,1.337$ & 1.115 & & $0.865,1.438$ \\
\hline Household income level & & & & & \\
\hline IPR $<130 \%$ (ref.) & & & & 1.000 & \\
\hline $130 \% \leq \mathrm{IPR}<300 \%$ & 1.034 & $0.837,1.277$ & 1.087 & & $0.857,1.380$ \\
\hline IPR $\geq 300 \%$ & 1.203 & $0.912,1.586$ & $1.254^{*}$ & & $1.005,1.565$ \\
\hline Obesity status & & & & & \\
\hline Non-obese $\left(\mathrm{BMI}<30.0 \mathrm{~kg} / \mathrm{m}^{2}\right)$ (ref.) & & & & 1.000 & \\
\hline Obese $\left(\mathrm{BMI} \geq 30.0 \mathrm{~kg} / \mathrm{m}^{2}\right)$ & 1.076 & $0.860,1.347$ & 0.930 & & $0.805,1.074$ \\
\hline Smoking status & & & & & \\
\hline Never smoker (ref.) & & & & 1.000 & \\
\hline Former or current smoker & 0.828 & $0.699,0.980$ & 0.972 & & $0.839,1.125$ \\
\hline Current health status & & & & & \\
\hline Fair or poor health (ref.) & & & & 1.000 & \\
\hline Good or excellent health & $1 \cdot 365^{\star}$ & $1.050,1.775$ & $1 \cdot 319^{\star \star}$ & & $1.090,1.595$ \\
\hline Health insurance status & & & & & \\
\hline Without health insurance (ref.) & & & & 1.000 & \\
\hline With health insurance & $1.430^{\star *}$ & $1.177,1.736$ & $1.222^{\star *}$ & & $1.055,1.415$ \\
\hline Chronic condition & & & & & \\
\hline No arthritis (ref.) & & & & 1.000 & \\
\hline Arthritis & 1.065 & $0.897,1.264$ & $1 \cdot 150$ & & $0.993,1.333$ \\
\hline No coronary artery disease (ref.) & & & & 1.000 & \\
\hline Coronary artery disease & $1 \cdot 712^{*}$ & $1 \cdot 016,2 \cdot 886$ & 1.404 & & $0.962,2.049$ \\
\hline No stroke (ref.) & & & & 1.000 & \\
\hline Stroke & $0.560^{*}$ & $0.352,0.891$ & 0.782 & & $0.533,1.148$ \\
\hline No cancer (ref.) & & & & 1.000 & \\
\hline Cancer & 1.040 & $0.787,1.375$ & 1.043 & & $0.798,1.362$ \\
\hline Survey wave & & & & & \\
\hline 2005-2006 (ref.) & & & & 1.000 & \\
\hline $2007-2008$ & $1.740^{\star \star *}$ & $1.480,2.046$ & $0.740^{* *}$ & & $0.607,0.902$ \\
\hline 2009-2010 & $2 \cdot 498^{\star \star \star}$ & $2.061,3.029$ & $1 \cdot 101$ & & $0.949,1.278$ \\
\hline
\end{tabular}

NHANES, National Health and Nutrition Examination Survey; IPR, income to poverty ratio; ref., reference category.

Individual-level data ( $n$ 5110) from NHANES 2005-2010 waves. Any use of nutrition facts label is defined as using labels sometimes, mostly or always. Regular use of nutrition facts label is defined as mostly or always using labels. Logistic regressions were conducted to estimate the odds ratios of nutrition facts label use among US adults, accounting for NHANES sampling design.

${ }^{\star} P<0.05,{ }^{\star *} P<0.01,{ }^{* \star} P<0.001$.

\section{Discussion}

Drawing from the health belief model, a teachable moment is a cueing event that increases perceptions of risk and outcome expectancies, prompts strong emotional responses and causes a redefinition of an individual's self-concept or social role ${ }^{(10,11)}$. According to McBride et al. ${ }^{(11)}$, a disease diagnosis is considered a teachable moment because it may 
Table 4 Adjusted prevalence of nutrition facts label use by diabetes status in NHANES 2005-2010 adult participants

\begin{tabular}{|c|c|c|c|c|}
\hline \multirow[b]{3}{*}{ Diabetes status } & \multicolumn{4}{|c|}{ Nutrition facts label use status } \\
\hline & \multicolumn{2}{|c|}{ Any use } & \multicolumn{2}{|c|}{ Regular use } \\
\hline & $\%$ & $95 \% \mathrm{Cl}$ & $\%$ & $95 \% \mathrm{Cl}$ \\
\hline No prediabetes/diabetes & 74.40 & $72.89,75.92$ & $39 \cdot 21$ & $37.46,40.96$ \\
\hline Undiagnosed prediabetes & 72.83 & $68.06,77.59$ & 39.95 & $34.02,45.89$ \\
\hline Undiagnosed diabetes & 71.50 & $59.64,83.37$ & 32.88 & $19 \cdot 11,46 \cdot 65$ \\
\hline Diagnosed prediabetes & $81 \cdot 16$ & $75 \cdot 27,87.06$ & $45 \cdot 28$ & $37 \cdot 28,53 \cdot 29$ \\
\hline Diagnosed diabetes & 85.93 & $82.91,88.95$ & $55 \cdot 60$ & $50 \cdot 04,61 \cdot 16$ \\
\hline
\end{tabular}

NHANES, National Health and Nutrition Examination Survey.

Individual-level data ( $n$ 5110) from NHANES 2005-2010 waves. Any use of nutrition facts label is defined as using labels sometimes, mostly or always. Regular use of nutrition facts label is defined as mostly or always using labels. Logistic regressions were conducted to estimate the adjusted prevalence of nutrition facts label use among US adults, accounting for NHANES sampling design.

increase one's perceptions of vulnerability and shift expectancies of and value assigned to health outcomes in relation to a specific unhealthy behaviour, bring out strong negative or positive emotional reactions, and alter perceived social norms or self-concept so that an unhealthy behaviour becomes less tolerable. The present study is the first attempt to evaluate the role of diabetes diagnosis as a potential teachable moment in nutrition facts label use. Using data from NHANES 2005-2010 waves, we found that people with diagnosed diabetes/prediabetes were substantially more likely to report nutrition facts label use in their daily grocery shopping compared with those with undiagnosed diabetes/prediabetes, whereas the pattern of nutrition facts label use appeared similar between people with undiagnosed diabetes/prediabetes and those without diabetes/prediabetes. These findings were consistent with our hypothesis based on the notion of teachable moment, which predicted increased efforts to seek healthier diet options following a diabetes diagnosis.

Self-reported use of nutrition facts label may not reflect actual use and translate into healthier grocery shopping behaviour or improved diet quality. Although a majority of US consumers report regular use of nutrition facts labels, in-store observations suggest actual use during food purchase can be lower ${ }^{(29)}$. The use of nutrition facts labels varies noticeably across population subgroups. Children, adolescents, obese older adults, individuals with less education and/or nutrition knowledge, people with lower disposable income and those with lower health awareness are less likely to use labels and/or effectively process the nutrition information presented ${ }^{(30-32)}$. Moreover, whether consumers can understand and use nutrition facts labels is contingent upon the purpose of the $\mathrm{ask}^{(5-8)}$. A majority appears capable to retrieve basic facts and make simple calculations/comparisons between products using numerical information on the label, but their ability and accuracy decline as the complexity of the task increases. Recent systematic reviews on nutrition labelling found that most studies focused on the use of labels to retrieve nutrition information and assess the healthiness of food products, whereas much less is known on whether and to what extent nutrition facts labels modify consumers' grocery shopping and dietary habits ${ }^{(4-8)}$. Elfassy et al. examined the association between hypertension and nutrition facts label use for sodium information among New York City adults ${ }^{(33)}$. Although individuals with hypertension had $71 \%$ higher odds of frequently using nutrition facts labels for sodium information compared with those with no hypertension, sodium intake was not found to differ by frequency of nutrition facts label use among those with hypertension. A vast majority of existing studies used a cross-sectional survey and/or qualitative study design, so that an estimated association between nutrition facts label use and healthier diet is subject to confounding (e.g. people who value health more are more likely to read nutrition facts labels and also more likely to purchase healthier foods).

A few limitations of the present study should be noted. The evidence indicating the impact of diabetes diagnosis on nutrition facts label use is at best preliminary and should not be interpreted as a causal relationship. The cross-sectional study design precludes examining life events and behaviours by chronological order, so that we do not know whether a modification in nutrition facts label use precedes or follows a diabetes diagnosis. Moreover, people with diagnosed diabetes/prediabetes could be different from those with undiagnosed diabetes/ prediabetes in disease history, severity, co-morbidity, etc. and these differences might confound the relationship between diabetes diagnosis and nutrition facts label use. Approximately $34 \%$ of the NHANES sample was excluded from the analysis due to missing values on test results, nutrition facts label use status and/or other individual characteristics. If those who did not report nutrition facts label use were less aware of nutrition labelling compared with those who reported label use, and those who did not undertake FPG and HbA1c testing were more or less likely to have undiagnosed diabetes/prediabetes compared with their counterparts who took the tests, respectively, we could underestimate or overestimate the impact of diabetes diagnosis on nutrition facts label use. Despite relatively large total sample size, undiagnosed diabetes 
cases ( $n$ 106) and diagnosed prediabetes cases ( $n$ 330) only occupied $0.86 \%$ and $4.06 \%$ of the sample, respectively. This might compromise model estimation precision and partially explain the lack of statistical significance in the difference of adjusted prevalence of regular nutrition facts label use between those with diagnosed prediabetes and those with undiagnosed prediabetes. The time span of patients living with diabetes/prediabetes might play a role in their nutrition facts label use (e.g. lack of novelty over time or sustained behaviour change after diagnosis), but such data were not available in the NHANES. Physical activity level could confound in the estimated relationship between diabetes diagnosis and nutrition facts label use. However, due to the measurement inconsistency in physical activity across the three waves of NHANES, we were not able to control for it in regression analysis. Finally, NHANES is a probability sample of the US noninstitutionalized population and patients in penal/mental facilities, institutionalized older adults and/or military personnel on active duty are not represented.

The prevalence of nutrition facts label use among people with diagnosed diabetes/prediabetes was substantially higher than that among people with undiagnosed diabetes/prediabetes, indicating a new diabetes diagnosis could serve as a window of opportunity for nutrition education and healthy diet intervention. Targeting people with newly diagnosed diabetes may augment intervention effectiveness as they could be particularly receptive to change ${ }^{(34)}$. Moreover, these people often can be conveniently reached at clinics, hospitals and other health-care settings when attending appointments for their new diagnosis. Health-care providers may seize this opportunity to offer on-site health behaviour counselling (e.g. nutrition and physical activity education, smoking cessation) shortly after delivering the diagnosis/treatment. Even a brief information session by health-care providers can lead to positive behaviour change ${ }^{(35-37)}$. This is particularly important given the fact that adults with chronic diseases currently are inadequately advised by health-care providers to adopt a healthier lifestyle ${ }^{(38-42)}$.

\section{Conclusion}

In conclusion, the present study examined the relationship between diabetes diagnosis status and nutrition facts label use among US adults aged 20 years and above using data from a nationally representative survey. People with diagnosed diabetes/prediabetes were noticeably more likely to report nutrition facts label use when making daily food purchase decisions relative to those with undiagnosed diabetes/prediabetes. In contrast, the prevalence of selfreported nutrition facts label use was similar between people with undiagnosed diabetes/prediabetes and those without diabetes/prediabetes. Serving as a potential teachable moment, diabetes diagnosis may positively impact nutrition facts label use and motivate diabetic patients to manage their condition through making healthier food choices. Future research needs to examine the potential dose-response relationship between frequency of nutrition facts label use and FPG/HbA1c level, the pathway linking nutrition facts label use motivated by diabetes diagnosis to actual food purchase and dietary intake, and explore optimal strategies to deliver nutrition interventions in conjunction with diabetes diagnosis/treatment in order to capitalize the benefit from this teachable moment.

\section{Acknowledgements}

Financial support: This research received no specific grant from any funding agency in the public, commercial or not-for-profit sectors. Conflict of interest: The author has no funding source or conflict of interest to declare. Authorship: R.A. designed the study, conducted statistical analysis and wrote the manuscript. Ethics of buman subject participation: NHANES was approved by the National Center for Health Statistics Research Ethics Review Board. The current analysis used NHANES de-identified public data and was deemed exempt by the University of Illinois at Urbana-Champaign Institutional Review Board.

\section{References}

1. van Dieren S, Beulens JW, van der Schouw YT et al. (2010) The global burden of diabetes and its complications: an emerging pandemic. Eur J Cardiovasc Prev Rehabil 17, Suppl. 1, 3-8.

2. Centers for Disease Control and Prevention (2014) National diabetes statistics report, 2014. Estimates of diabetes and its burden in the United States. http://www.cdc.gov/diabetes/ pdfs/data/2014-report-estimates-of-diabetes-and-its-burdenin-the-united-states.pdf (accessed March 2015).

3. Evert $\mathrm{AB} \&$ Boucher JL, American Diabetes Association et al. (2013) Nutrition therapy recommendations for the management of adults with diabetes. Diabetes Care 36, 3821-3842.

4. Campos S, Doxey J \& Hammond D (2011) Nutrition labels on pre-packaged foods: a systematic review. Public Health Nutr 14, 1496-1506.

5. Drichoutis AC, Lazaridis P \& Nayga RM Jr (2006) Consumers' use of nutritional labels: a review of research studies and issues. Acad Mark Sci Rev 9, 93-118.

6. Mhurchu CN \& Gorton D (2007) Nutrition labels and claims in New Zealand and Australia: a review of use and understanding. Aust N Z J Public Health 31, 105-112.

7. Cowburn G \& Stockley L (2005) Consumer understanding and use of nutrition labelling: a systematic review. Public Health Nutr 8, 21-28.

8. Grunert KG \& Wills JM (2007) A review of European research on consumer response to nutrition information on food labels. J Public Health 15, 385-399.

9. American Diabetes Association (2014) Taking a closer look at labels. http://www.diabetes.org/food-and-fitness/food/ what-can-i-eat/food-tips/taking-a-closer-look-at-labels.html (accessed March 2015).

10. Lawson PJ \& Flocke SA (2009) Teachable moments for health behavior change: a concept analysis. Patient Educ Couns 76, 25-30. 
11. McBride CM, Emmons KM \& Lipkus IM (2003) Understanding the potential of teachable moments: the case of smoking cessation. Health Educ Res 18, 156-170.

12. Demark-Wahnefried W, Aziz NM, Rowland JH et al. (2005) Riding the crest of the teachable moment: promoting long-term health after the diagnosis of cancer. J Clin Oncol 23, 5814-5830.

13. Hite D (2011) Teachable moments in diabetes care: approaching patients at the point of diagnosis. http://www.diabetesproductsource.com/blog/teachablemoments-diabetes-care-approaching-patients-pointdiagnosis (accessed March 2015).

14. McBride CM \& Ostroff JS (2003) Teachable moments for promoting smoking cessation: the context of cancer care and survivorship. Cancer Control 10, 325-333.

15. Mills AL \& Pierce JP (2008) Using teachable moments to improve nutrition and physical activity in patients. Am Fam Physician 77, 1510-1511.

16. Falba T (2005) Health events and the smoking cessation of middle aged Americans. J Behav Med 28, 21-33.

17. Karlsen RV, Bidstrup PE, Christensen J et al. (2006) Men with cancer change their health behaviour: a prospective study from the Danish diet, cancer and health study. $\mathrm{Br} \mathrm{J}$ Cancer 107, 201-206.

18. Keenan PS (2009) Smoking and weight change after new health diagnoses in older adults. Arch Intern Med 169, 237-242.

19. Margolis R (2013) Educational differences in healthy behavior changes and adherence among middle-aged Americans. J Health Soc Behav 54, 353-368.

20. Newsom JT, Huguet N, McCarthy MJ et al. (2012) Health behavior change following chronic illness in middle and later life. J Gerontol B Psychol Sci Soc Sci 67, 279-288.

21. Nothwehr F \& Stump T (2000) Health-promoting behaviors among adults with type 2 diabetes: findings from the Health and Retirement Study. Prev Med 30, 407-414.

22. van Gool CH, Kempen GI, Penninx BW et al. (2003) Relationship between changes in depressive symptoms and unhealthy lifestyles in late middle aged and older persons: results from the Longitudinal Aging Study Amsterdam. Age Ageing 32, 81-87.

23. Twardella D, Loew M, Rothenbacher D et al. (2006) The diagnosis of a smoking-related disease is a prominent trigger for smoking cessation in a retrospective cohort study. J Clin Epidemiol 59, 82-89.

24. Wray LA, Herzog AR, Willis RJ et al. (1998) The impact of education and heart attack on smoking cessation among middle-aged adults. J Health Soc Behav 39, 271-294.

25. Bidstrup PE, Dalton SO, Christensen J et al. (2013) Changes in body mass index and alcohol and tobacco consumption among breast cancer survivors and cancer-free women: a prospective study in the Danish Diet, Cancer and Health Cohort. Acta Oncol 52, 327-335.

26. Hawkes AL, Lynch BM, Youlden DR et al. (2008) Health behaviors of Australian colorectal cancer survivors, compared with noncancer population controls. Support Care Cancer 16, 1097-1104.
27. Williams K, Steptoe A \& Wardle J (2013) Is a cancer diagnosis a trigger for health behaviour change? Findings from a prospective, population-based study. $\mathrm{Br} J$ Cancer 108, 2407-2412.

28. Li Y, Geiss LS, Burrows NR et al. (2013) Awareness of prediabetes - United States, 2005-2010. MMWR Morb Mortal Wkly Rep 62, 209-212.

29. Azman N \& Sahak SZ (2014) Nutritional label and consumer buying decision: a preliminary review. Procedia Soc Behav Sci 130, 490-498.

30. Satia JA, Galanko JA \& Neuhouser ML (2005) Food nutrition label use is associated with demographic, behavioral, and psychosocial factors and dietary intake among African Americans in North Carolina. J Am Diet Assoc 105, 392-402.

31. Govindasamy R \& Italia J (2000) The influence of consumer demographic characteristics on nutritional label usage. J Food Prod Mark 5, 55-68.

32. Grunert KG, Wills JM \& Fernández-Celemín L (2010) Nutrition knowledge, and use and understanding of nutrition information on food labels among consumers in the UK. Appetite 55, 177-189.

33. Elfassy T, Yi S, Eisenhower D et al. (2015) Use of sodium information on the nutrition facts label in New York City adults with hypertension. $J$ Acad Nutr Diet 115, 278-283.

34. Xiang X (2015) Adoption and maintenance of health behaviors among middle-aged and older adults: the role of chronic disease diagnosis and depression. PhD Thesis, University of Illinois at Urbana-Champaign.

35. Bull FC \& Jamrozik K (1998) Advice on exercise from a family physician can help sedentary patients to become active. Am J Prev Med 15, 85-94.

36. Glasgow RE, Eakin EG, Fisher EB et al. (2001) Physician advice and support for physical activity: results from a national survey. Am J Prev Med 21, 189-196.

37. Greenlund KJ, Giles WH, Keenan NL et al. (2002) Physician advice, patient actions, and health-related quality of life in secondary prevention of stroke through diet and exercise. Stroke 33, 565-570.

38. Fallon EA, Wilcox S \& Laken M (2006) Health care provider advice for African American adults not meeting health behavior recommendations. Prev Chronic Dis 3, A 45 .

39. Lobo IE, Loeb DF, Ghushchyan V et al. (2012) Missed opportunities for providing low-fat dietary advice to people with diabetes. Prev Chronic Dis 9, E161.

40. Morrato EH, Hill JO, Wyatt HR et al. (2006) Are health care professionals advising patients with diabetes or at risk for developing diabetes to exercise more? Diabetes Care 29, 543-548.

41. Nguyen HT, Markides KS \& Winkleby MA (2011) Physician advice on exercise and diet in a US sample of obese Mexican-American adults. Am J Health Promot 25, 402-409.

42. Xiang X, Hernandez R \& Larrison CR (2015) Provider advice on exercise and diet among adults with comorbid serious psychological distress and diabetes or diabetes risk factors. Diabetes Educ 41, 185-193. 